\title{
Les apports de l'instrumentation nucléonique et des traceurs à la sédimentologie dynamique
}

\author{
P. Brisset, A. Vabre, A. Poggi \\ Commissariat à l'Energie Atomique - DTA - DAMRI - SAR - Centre de Saclay - BP 52 - \\ 91193 Gif sur Yvette Cedex
}

\section{Résumé}

La mécanique des fluides appliquée aux transports sédimentaires est souvent mise en défaut, en particulier lorsque le déplacement des matériaux s'effectue par charriage.

Les traceurs sont un moyen particulièrement attrayant de mesure directe qui a permis ces dernières décennies de faire évoluer les connaissances de la sédimentologie dynamique appliquée au génie civil où les aspects industriels, économiques et environnementaux sont étroitement liés. Les premières expériences avec des traceurs, radioactifs, pour étudier les mouvements sédimentaires naturels datent de 1954. Depuis, des développements méthodologiques et technologiques (nouveaux traceurs radioactifs et traceurs non radioactifs) importants ont permis d'élargir leur domaine d'action.

A côté des traceurs se sont développés des instruments basés sur l'interaction des rayonnements provenant d'une source scellée avec la matière et permettant de mesurer directement in situ la concentration du matériau au point de mesure ou son épaisseur. Ces appareils sont utilisés essentiellement dans le domaine de l'optimisation et de la gestion des travaux de dragage et ont permis de réaliser des économies importantes en draguant mieux et moins. La réduction des quantités draguées a également un impact sur l'environnement.

\begin{abstract}
The fluid mechanics applied to sediment transport provides in many cases bad results, and especially for bed-load transport .

The tracers are a very attractive direct measurement mean which has allowed, during the last decades, important evolutions in the knowledge on sediment transpont applied to civil engineering when environmental, industrial and economical aspects are strongly connected . The first experiment with radioactive tracers has been carried out in 1954 . Since this time, the tracers have increased their field of action through important methodological and technological developments .

Beside the tracers, the nucleonic control systems (instruments using the interaction of gamma rays from a sealed source with the matter ) give the possibility to measure directly in situ either a sediment concentration of sediments in water or sediments layer thickness. These tools are mainly used in the management and optimisation of dredging works field of activities and have allowed important cost savings in dredging better and less, so with a lower impact on the environment.
\end{abstract}




\section{Vímes Journées Nationales Génie Civil - Génie Côtier, Caen, France, 17-19 Mai 2000}

Bien qu'ayant une grande importance économique en raison du coût des ouvrages et des travaux qu'ils imposent pour lutter contre leurs nombreuses nuisances (comblement des retenues, difficultés de fonctionnement des dispositifs évacuateurs, impact des opérations de vidange, érosion du littoral, travaux de dragage, rejet de toutes sortes dans le milieu aquatique de polluants particuliers, etc...), les transports sédimentaires sous l'action des courants et des houles sont mal connus. Même en faisant appel aux théories les plus élaborées, la mécanique des fluides est encore actuellement impuissante à résoudre les problèmes posés en utilisant uniquement des équations : l'expérimentation et les mesures doivent toujours intervenir à un moment ou à un autre, en nature et en modèle physique. Or le manque de moyens de mesure entrave la réflexion sur les processus physiques des transports solides et, a fortiori, sur l'estimation de leur intensité.

C'est pourquoi les techniques nucléaires (traceurs radioactifs et jauges nucléaires. ) se sont progressivement perfectionnées au fil des années, car elles offrent, grâce aux propriétés spécifiques de la radioactivité, des avantages piécieux:

- mesures directes des traceurs en nature et en modèle physique,

- mesures lagrangiennes des déplacements,

- mesure et suivi sur une faible proportion ( traceurs ) du contenu sédimentaire donc rapidité d'auscultation des phénomènes

- mesures en continu des turbidités, même au travers d'une paroi opaque.

- Etc.....

Ces techniques se sont développées, sans suivre un programme rigoureux mais de manière pragmatique, en essayant d'anticiper et de satisfaire les demandes d'utilisateurs: ports français et étrangers, laboratoires d'hydraulique, directions départementales d'équipement, universités, service technique central de la direction des ports maritimes, IFREMER, mais aussi des organismes internationaux comme l'Agence Internationale de l'Energie Atomique ou l'International Water Management Institute.

Aujourd'hui, à côté des traceurs radioactifs se développent d'autres techniques complémentaires de traçage, non radioactifs (fluorescents, radioactivables, magnétiques,...) aux performances différentes, permettant de répondre à des problématiques où les traceurs radioactifs sont difficilement envisageables (généralement pour des raisons psychologiques, quelquefois pour des raisons techniques et réglementaires).

\section{Les traceurs}

\subsection{Principe}

La notion de traceur appartient au sens commun: dans la zone à étudier, on introduit un sédiment marqué par un élément ayant une propriété particulière mesurable. Puis au moyen de détecteurs appropriés, ou par prélèvement, on détermine les déplacements du nuage formé par les particules marquées en fonction de l'espace et du temps. Les résultats quantitatifs (Sauzay, 1967) sont ensuite interprétés en fonction des paramètres hydrométéorologiques enregistrés par ailleurs. Les informations recueillies sont globales (on dit que la méthode est intégratrice) et généralement il n'est pas possible de déterminer les paramètres fondamentaux tels que la vitesse critique d'entrainement ou la rugosité des fonds, etc... 


\subsection{Les traceurs radioactifs}

\subsubsection{Méthodologie}

Les spécialistes disposent ( Caillot, 1983) d'un grand nombre de radionucléides qui leur permettent d'aborder différents problèmes sédimentologiques (en nature ou en laboratoire ) qui concernent aussi bien les particules dans une gamme très large ( de la vase aux galets. Le tableau 1 en donne les principales caractéristiques ainsi que les activités généralement mises en jeu lors de la réalisation de ces expérimentations. Ces activités sont du reste toujours très inférieures aux activités maximales autorisées.

D'une manière schématique, on peut dire que :

- les galets, supérieurs à $5 \mathrm{~cm}$ de diamètre, sont marqués individuellement par une source radioactive formée par un fil métallique : iridium 192 , tantale 182 ou argent 110.

- les sables, de 0,040 à $2,5 \mathrm{~mm}$ de diamètre, sont simulés par des verres spéciaux ayant une densité de l'ordre de 2,65. Leur distribution granulométrique est choisie identique à celle des sédiments naturels en place sur le site ou comme une fraction représentative de ceux-ci. Ces verres ainsi préparés sont rendus radioactifs après un court séjour dans un réacteur nucléaire.

- les vases ou sédiments cohésifs inférieurs à $40 \mu \mathrm{m}$ de diamètre sont marqués directement par chimisorption au moyen de solutions radioactives. Les réactions physico-chimiques sont sélectionnées et conduites de telle sorte que le comportement hydrodynamique des sédiments ainsi marqués reste identique à celui des sédiments naturels, lorsque l'un et l'autre sont placés dans les mêmes conditions de transport ou de sédimentation.

Lors des expérimentations en laboratoire, il devient habituel d'utiliser des générateurs d'isotopes radioactifs, systèmes automatiques et protégés qui permettent d'obtenir aisément une solution radioactive au moment de son emploi. Ces dispositifs sont journellement employés en médecine nucléaire.

Les quantités nécessaires au bon déroulemcint d'une expérience de traceurs sont faibles : 200 galets par site; 0,25 à $1 \mathrm{~kg}$ de verre radioactif ; 5 à 15 litres de suspension de vase à $200 \mathrm{~g} / 1$ sont généralement suffisants. Cela facilite la manipulation des produits sur le site, leur immersion et surtout leur intégration rapide au milieu.

L'introduction de sédiments-traceurs peut s'effectuer soit par dépôt sur les fonds, soit par création d'un nuage de particules en suspension en simulant un rejet, soit par mélange avec des sédiments fins dans un puits de drague avant de procéder aux opérations de rejet.

Les détecteurs de rayonnement à scintillation de type océanographique sont à la fois sensibles et résistants. L'électronique de mesure embarquée est portative et autonome : ces ensembles permettent d'entreprendre des expériences dans des pays lointains et avec des moyens nautiques limités ce qui rend la méthode particulièrement robuste pour des applications terrain.

Ces capteurs sont placés à la remorque d'une embarcation de dimensions modestes (10 à $20 \mathrm{~m}$ de longueur) soit sur le fond (mesure de charriage), soit en suspension (étude des rejets). Dans ce dernier cas, les détecteurs sont associés à des capteurs de pression donnant la profondeur d'immersion. La position du bateau est relevée en continu par un système de radiolocalisation. 
1.'interprétation des mesures conduit à un lot de résultats qualitatifs et quantitatifs :

- Pour les déplacements sédimentaires sur les fonds :

- la ou les directions du mouvement,

- les vitesses maximales et moyennes des déplacements horizontaux,

- la quantité de sédiments transférés par charriage,

- la remise en suspension ou le recouvrement éventuel.

- Pour les déplacements sédimentaires de suspensions artificiellement rejetés dans le milieu (rejets industriels et urbains, produits de dragage) :

- l'excursion et la dérive ; direction, vitesse horizontale,

- les coefficients de dispersion longitudinaux et transversaux,

- le taux de dilution en fonction du temps et de la distance parcourue par le nuage.

- la vitesse moyenne de sédimentation des particules,

- la quantité de matière déposée sur les fonds.

\subsubsection{Quelques exemples d'utilisation}

La méthode des traceurs est utilisée en nature pour réaliser des études de site mais aussi des étuñes à caractère général.

\subsubsection{Etude systématique de sites}

- port et littoral de Zeebrugge (Belgique) ( Bastin, Caillot et al,1982)

- baie de Seine (Avoine, Caillot et al,1984)

- Cap Breton ( Gourlez, Castaing, 1985)

- Honfleur ( Hoslin, Brisset et al, 1989)

- etc......

2.2.2.2. Etude du rejet des produits de dragage : ports du Havre, d'Antifer, de Lorient, de Singapour, etc .......

2.2.2.3. Recyclage des produits de dragage : ports de Lorient, de Zeebrugge.

A Zeebrugge, les matériaux rejetés au large, à $18 \mathrm{~km}$ de la côte par des fonds de $-15 \mathrm{~m}$, reviennent à plus des $2 / 3$ et rapidement (moins de 100 jours) à la côte sous l'action des courants de marée puis participent au transit littoral avant de se redéposer dans les eaux calmes des bassins portuaires. Ce recyclage a été mis en évidence en utilisant des éléments radioactifs (hafnium et terbium) n'existant pas dans la nature. Certaines mesures, en laboratoire spécialisé ont été faites après une dilution de $10^{-14}$ (Brisset, Hoslin et al, 1995) 
2.2.2.4.Etudes présentant un caractère général

- étude du mécanisme bouchon vaseux - crème de vase dans un estuaire. L'expérience en vraie grandeur prouve la circulation dans le temps et l'espace de la masse sédimentaire dans un estuaire (Hoslin, 1980)

- étude de l'action de la houle sur les transports sédimentaires.

\subsubsection{Transports solides en régime torrentiel}

Ce type de transport très complexe fait intervenir un trop grand nombre de paramètres pour pouvoir être traité uniquement par les modèles mathématiques. Aussi le nombre d'expériences réalisées dans ce domaine est important (Tola, 1989).

Parmi celles-ci :

- dans les Pyrénées orientales: Verdouble, Cady, Tet, Lentilla, Agly,

- dans les Vosges : Bruche, Mossig, Doller,

- en Corse : Fium'Orbo.

La méthode est aussi utilisée, avec un grand succès, sur les cordons littoraux de galets.

\subsubsection{Etude des rejets sous forme particulaire}

- Analyse systématique des rejets urbains en mer sous forme particulaire (matières organiques et argileuses) contaminés par des polluants chimiques et biologiques.

- Etude de la pollution mécanique des rivières (Doubs) par les fines particules remises en suspension par le dragage. Ce travail a rendu possible des recommandations sur les conditions d'extractions des sables et graviers (Brisset , 1986)

\subsubsection{Emploi de traceurs radioactifs en modèle physique et canal de laboratoire}

Les premières théories et techniques expérimentales d'estimation du transport solide dans les écoulements à surface libre sont basées sur la méthode d'Euler : mesure d'une quantité en point fixe en fonction du temps. Or le transport permanent de la couche supérieure du lit sédimentaire est le résultat d'une série alternée de sauts et de périodes de repos des grains de sable qui dépendent des forces hydrodynamiques instantanées et imprévisibles caractéristiques $\mathrm{d}^{\prime 3}$ un processus aléatoire. Les traceurs radioactifs en permettant des mesures lagrangiennes se sont révélés très utiles pour déterminer les trajectoires des particules isolées et des groupes de particules tout au long d'un canal. D'autres travaux également en canal de laboratoire ont permis de déterminer (Wilson, 1987) simultanément le débit solide par charriage et le débit solide en suspension. Récemment des traceurs ont été utilisés pour étudier en canal les mécanismes de rejets de produits de dragage (Boutin, 1999). Dans tous ces cas les traceurs radioactifs jouent un rôle prépondérant et, semble-t-il, irremplaçable en raison de leur grande sensibilité de mesure. 


\subsubsection{Commentaires}

Le grand nombre d'études réalisées, les larges possibilités offertes et la souplesse de cette méthode plaident en sa faveur. L'expérience acquise montre que ces procédés fournissent une bonne estimation des quantités de sédiments transportés avec, dans le pire des cas, une incertitude de 50 à $100 \%$ : ce qui est peu pour des mesures en nature et lorsqu'on sait qu'il y a souvent un facteur 10 d'écart (et parfois plus!) entre les diverses formules empiriques d'estimation du débit solide à l'équilibre. Cependant, la technique des traceurs reste limitée dans l'espace et le temps: le kilomètre carré, quelques mois (au plus six mois) et par l'impossibilité physique de détecter les radionucléides utilisés au-delà d'une profondeur d'enfouissement de $0,8 \mathrm{~m}$. Récemment, dans certaines circonstances, ces limites ont été largement repoussées en particulier en terme de distance : quelques dizaines de $\mathrm{km}$ en milieu marin .

Remarque : les manipulations sérieuses et bien conçues ont toujours été autorisées depuis 1954 par la Commission Interministérielle des Radioéléments Artificiels ( CIREA) après examen d'un dossier circonstancié.

\subsection{Les traceurs non radioactifs}

Plusieurs types de traceurs non radioactifs existent ou sont en cours de développement:

- fluorescents : ces traceurs concernent essentiellement les particules sableuses. Une détection in situ est possible sur estran. Dans les autres cas, des prélèvements suivis de mesures en laboratoire sont indispensables. Quelques développements technologiques sont en cours. Un premier système est opérationnel et permet, sur prélèvement, de dénombrer les grains de traceur et de déterminer la courbe granulométrique de l'échantillon et du traceur. D'autres systèmes en cours de développement devraient permettre une mesure in situ sous l'eau.

- radioactivables: le principe consiste à marquer les sédiments (vases ou sables) par un élément activable en réacteur. L'élément activable doit être choisi en fonction des éléments traces présents naturellement sur le site d'étude. Les mesures se font par prélèvement puis activation en réacteur et enfin analyse de la teneur en élément traceur dans l'échantillon par spectrométrie gamma bas bruit de fond.

- magnétiques : ces traceurs sont en cours de développement tant au niveau des traceurs euxmêmes que des moyens de détection. Plusieurs principes sont envisageables (RPE, susceptibilité magnétique, magnétisme total,...).

Les traceurs fluorescents et activables ont été récemment utilisés conjointement pour une étude concernant les transferts de solides dans les grands réseaux d'irrigation au Pakistan ( Vabre, 1998) ( Vabre, 1999 )

\section{Les capteurs radiométriques}

\subsection{Principales caractéristiques}

Un capteur radiométrique ou jauge nucléaire est l'association optimale d'une source radioactive scellée et d'un détecteur de rayonnement adapté. L'interaction rayonnement nucléaire-matière examinée est mise à profit pour mesurer en continu la concentration ou la densité de sédiments en suspension ou déposés, cela permet d'obtenir des mesures non destructives, sans faire de prélèvements et en continu même à travers une paroi opaque ou directement dans l'eau. Ces informations sont traitées en temps réel grâce à un 
microcalculateur. Elles sont ainsi devenues une aide à la décision et à la gestion d'un ouvrage ou d'une installation.

Les spécialistes distinguent deux types de dispositifs :

- les jauges à transmission,

- les jauges à diffusion.

\subsection{Exemples de jauges nucléaires}

\subsubsection{Les appareils de terrain}

- mesure de turbidité directement in situ ou à travers une canalisation (Meyer,1989). Le dispositif est d'autant plus performant que la concentration en sédiment est supérieure à $1 \mathrm{~g} / \mathrm{l}$.

- mesure du profil vertical de densité de matériaux déposés dans les chenaux envasés, puits de drague et retenues de barrage ( Meyer, Meinard et al, 1986)

Les informations obtenues, complémentaires des sondeurs à ultrasons qui ne localisent que l'interface eau-vase liquide, permettent de repousser la limite des profondeurs navigables: soit, dans un chenal classique de navigation, une économie de plusieurs MF par an ..

- pilotage de la vidange des retenues de barrage (Meyer, Brisset et al, 1998)

- mesure des variations de hauteur des formes sédimentaires et de la direction du transport

(Brisset et al, 2000)

\subsubsection{Les appareils de laboratoire}

- mesure non invasive et non destructive du profil de densité d'une carotte sédimentaire.

- mesure du gradient de tassement des sédiments fins en fonction du temps, de la hauteur du culot, de la concentration initiale de la suspension, de la hauteur du volume de sédimentation.

\subsection{Commentaires}

Tous ces dispositifs respectent les normes d'irradiation au contact. Ils sont également conçus pour être utilisés par un personnel non directement affecté au travail sous rayonnement ionisant. Cependant le propriétaire doit être autorisé par la Commission Interministérielle des Radioéléments Artificiels (CIREA) à détenir et à utiliser des radioéléments sous forme de sources, ce qui implique sa compétence.

Le plus souvent, la précision de ces appareils est de l'ordre de $1 \%$ absolu avec un degré de confiance de $68 \%$. Ces instruments sont d'autant plus performants et sans concurrents, que la concentration est élevée. En dessous de $1 \mathrm{~g} / \mathrm{l}$, il est nécessaire de faire appel à d'autres procédés.

\section{Conclusions}

L'industrie, les besoins de la navigation et le tourisme ont parfois tendance à exploiter et à domestiquer avec plus ou moins de brutalité les fleuves, les estuaires, le littoral. Le recul des côtes, l'extraction des granulats, les rejets des eaux usées et des produits de dragage, les vidanges de retenues de barrage, etc..., sont des problèmes d'actualités demandant une connaissance précise des mécanismes de transport, de dilution et de sédimentation et la mesure des paramètres qui régissent ces processus afin de définir la meilleure gestion possible des milieux fluviaux, estuairiens et côtiers. 
Les traceurs et les jauges nucléaires sont actuellement l'un des rares moyens, éprouvés par de nombreuses etudes, qui permettent aux ingénieurs, aux hydrauliciens et aux chercheurs de disposer des informations et des mesures dont ils ont de plus en plus impérativement besoin.

Six principaux domaines, où les impacts économiques et écologiques sont considérables, devraient voir leur développement se confirmer et s'intensifier :

- Gestion des travaux de dragage : rejet des produits de dragage-navigabilité dans les chenaux envasés.

2) Stabilité du littoral : action de la houle et des courants sur les transports sédimentaires, extraction en mer de granulats

(3 Rejets urbains et industriels sous formes particulaires dans les estuaires et en mer.

4 Mesure en continu des MES, en particulier dans les stations de traitement des eaux.

$\boldsymbol{6}$ Etude de gestion des vidanges des retenues de barrage.

Au moment où les frontières européennes s'ouvrent, ces techniques pourraient permettre aux ingénieurs français du génie civil ayant en charge les problèmes posés par les transports solides de proposer des procédés originaux performants et d'avant garde par rapport à leurs concurrents qui le plus souvent ne disposent pas de cet atout complémentaire.

\section{Références}

Avoine J., Caillot A., Hoslin R., Larsonneur C., Massias J., Quesbey M. - Mise en évidence des mouvements sédimentaires en baie de Seine à l'aide de traceurs radioactifs, contribution à l'étude du comblement de l'estuaire de la Seine - SHF, XVIII ${ }^{\circ}$ journées de l'hydraulique, rapport 12, Marseille, 1984

Bastin A., Caillot A., Malherbe B. - Zeebrugge port extension , Sediment transport measurement on and off the belgium coast by means of tracers - Engineering in marin environment, Brugges, 1982

Boutin R. - Amélioration des connaissances sur le comportement des rejets en mer de produits de dragage de type vase - Phénomènes à court terme et dans le champ proche, Thèse, INSA Lyon , 1999

Brisset P. - Etude de la pollution mécanique des rivières par les extractions de granulats, application au cas du Doubs, Thèse, Institut National polytechnique de Toulouse, 1986

Brisset P., Hoslin R., Meyer G., Caillot A. - Study and optimisation of dredging works utilizing radioactivity, the case of Zeebrugge harbour - Symposium on isotopes in water ressources management, AIEA, Vienna, 20-24 march, 1995

Brisset P., Meyer G. , Galpin J., Lamdasni Y. - Development of a measurement system to study sandwave dynamics through the absorption of scaterred gamma rays - International Workshop on marine sandwave dynamics, Lille, 2000

Caillot A. - Guide book on nuclear techniques in hydrology, Report series $\mathrm{n}^{\circ} 91$, chapitre 9 : bedload transport, p103 à 128, AIEA, 1983

Gourlez P., Castaing P. - Etude des mouvements sédimentaires le long du littoral de part et d'autre du gouf de Capbreton au moyen de traceurs radioactifs et fluorescents - C.R Bordomer du 8 au 11 novembre 1985 
Hoslin R. - Le double marquage de la vase par le zinc et l'hafnium... Fude du comportement du zinc et des mouvements sedimentaires dans le système Garonne-Gironde - Thèse , Faculté des Sciences Paris sud, 1980

Hoslin R. , Brisset P., Massias J., Poggi A, Sannié G. - Etude, au moyen de traceurs radioactifs de l'évolution des dépôts de dragage situés dans l'estuaire de la Seine, Zones du dépôt intermédiaire et du vidage aval - rapport ORIS/SAR/S/89-112/H56, 1989

Meyer G. - Les capteurs radiométriques en sédimentologie dynamique - La Houille Blanche $\mathrm{n}^{\circ} 3-4, \mathrm{p} 235$ à 238,1989

Meyer G., Meinard, Granboulan, Babylon - Etude de la décantation de sédiments fins dans le puits d'une drague aspiratrice en marche à l'aide d'une sonde nucléaire - Congrès mondial de dragage, Brighton , 1986

Meyer G., Brisset P., Hoslin R., Poggi A, - Protection de l'environnement au cours de chasses de barrage - Hydrological symposium on medelling soil erosion and closely related hydrological processes, AIEA, 13-17 juillet 1998

Sauzay G. - Methode du bilan des taux de comptage d'indicateurs radioactifs pour la détermination du débit de charriage de lits sableux - Thèse, Université de Toulouse, 1967

Tola F. - Etude du transport des galets par les torrents au moyen de traceurs radioactifs- La Houille Blanche $n^{\circ} 3-4$, p231 à 234, 1989

Vabre A. - Campagne de mesures au moyen de traceurs radioactivables et fluorescents sur Jamrao canal system ( Sindh, Pakistan ) - VIo joumées nationales Génie côtier-Génie civil, $17-19$ mai 2000

Wilson G. - Etude du transport et de la dispersion des sédiments en tant que processus aléatoire-Doctorat d'état ,Jussieu, 1986 


\section{TABLEAU 1}

Liste des radionucléides utilisés en sédimentologie

\begin{tabular}{|c|c|c|c|c|c|c|c|c|c|c|c|c|}
\hline \multicolumn{2}{|c|}{ Isotopes } & $\mid \begin{array}{l}{ }^{292 m} \mathrm{TC}^{(1)} \\
\text { recennetium }\end{array}$ & \begin{tabular}{|l}
${ }^{133} \mathrm{In}^{101}$ \\
indiumm
\end{tabular} & $\begin{array}{l}{ }^{198} \mathrm{Au} \\
\mathrm{Or}^{2}\end{array}$ & $\begin{array}{l}\text { Wh } \mathrm{Nd} \\
\text { NÉodyme }\end{array}$ & $\begin{array}{l}{ }^{51} \mathrm{Cr} \\
\text { Chrome }\end{array}$ & $\begin{array}{l}\begin{array}{l}170+181 \\
\text { Hafnium }\end{array} \\
\text { Hafn }\end{array}$ & $\begin{array}{l}{ }^{192} \mathrm{Ir} \\
\text { Iridium }\end{array}$ & $\begin{array}{l}{ }^{46} \mathrm{Sc} \\
\text { Scandium }\end{array}$ & $\begin{array}{l}{ }^{182} \mathrm{Ta} \\
\text { Tantale }\end{array}$ & ${ }_{\text {Zinc }} \mathrm{Zn}$ & ${ }_{\text {Atgen! }}^{110} \mathrm{Ag}$ \\
\hline \multicolumn{2}{|c|}{ Périodes } & 6 h 02 & $100 \mathrm{~min}$ & $2,7 \mathrm{j}$ & $11 \mathrm{j}$ & $27 \mathrm{j}$ & 40 et $70 j$ & $74 \mathrm{j}$ & $84 \mathrm{j}$ & $111 \mathrm{j}$ & $245 j$ & $253 j$ \\
\hline \multicolumn{2}{|c|}{$\begin{array}{l}\text { Energie des rayons } \gamma \\
\quad \text { (en Mev) }\end{array}$} & 0,14 & 0,39 & 0,41 & $\begin{array}{l}\text { Spectre } \\
\text { complexe }\end{array}$ & $\begin{array}{l}0,32 \\
(9 \% \\
\text { d'émission) }\end{array}$ & $\begin{array}{l}\text { Spectre } \\
\text { complexe }\end{array}$ & $\begin{array}{l}\text { Spectre } \\
\text { complex } \\
\mathrm{e}\end{array}$ & $\begin{array}{ll}0,9 & \text { et } \\
1,1 & \end{array}$ & $\begin{array}{l}\text { Spectre } \\
\text { complexe }\end{array}$ & $\begin{array}{l}\text { Spectre } \\
\text { complexe }\end{array}$ & $\begin{array}{l}\text { Spectre } \\
\text { complexe }\end{array}$ \\
\hline \multirow{3}{*}{$\begin{array}{l}\text { Forme } \\
\text { utilisation }\end{array}$} & Vases & (2) & (3) & $\mathrm{x}$ & & $\mathrm{x}$ & $\mathrm{x}$ & & $\mathrm{x}$ & & $\mathrm{x}$ & \\
\hline & Sables & & & $x$ & $\mathrm{x}$ & $x$ & & $\mathrm{x}$ & $\mathrm{x}$ & & & \\
\hline & Galets & & & & & & & $\mathrm{x}$ & - & $x$ & & $\mathrm{x}$ \\
\hline \multicolumn{2}{|c|}{$\begin{array}{l}\text { Activités } \\
\text { généralemnent utilisées }\end{array}$} & $\begin{array}{l}10 \\
\grave{a} \\
100 \mathrm{mC} \mathrm{f}^{(4)}\end{array}$ & $\begin{array}{l}1 \\
a \\
25 \mathrm{mCi}^{(4)}\end{array}$ & $\begin{array}{l}10 \mathrm{mCi}^{(4)} \\
5 \text { à } 9 \mathrm{Ci}^{(4)}\end{array}$ & 1 à $3 \mathrm{Ci}$ & 10 à $20 \mathrm{Ci}$ & 1 à $5 \mathrm{Ci}$ & 1 à $3 \mathrm{Ci}$ & 1 à $9 \mathrm{Ci}$ & $\begin{array}{l}0,1 \mathrm{mCi} \\
\text { par galet }\end{array}$ & 1 à $5 \mathrm{Ci}$ & $\begin{array}{l}0,1 \mathrm{mCi} \\
\text { par galet }\end{array}$ \\
\hline
\end{tabular}

Rappel : $1 \mathrm{Ci}=3,710^{10}$ Becquerel 